\title{
Statistical Description of Laser Damage Initiation in NIF and LMJ Optics at $355 \mathrm{~nm}$
}

M. D. Feit, F. Y. Génin, A. M. Rubenchik, L. M. Sheehan, S. Schwartz, M. R. Kozlowski, J. Dijon, P. Garrec, J. Hue

This paper was prepared for submittal to the Third Annual International Conference on Solid State Lasers for Application (SSLA) to Inertial Confinement Fusion (ICF) Monterey, California June 7-12, 1998

July 30, 1998

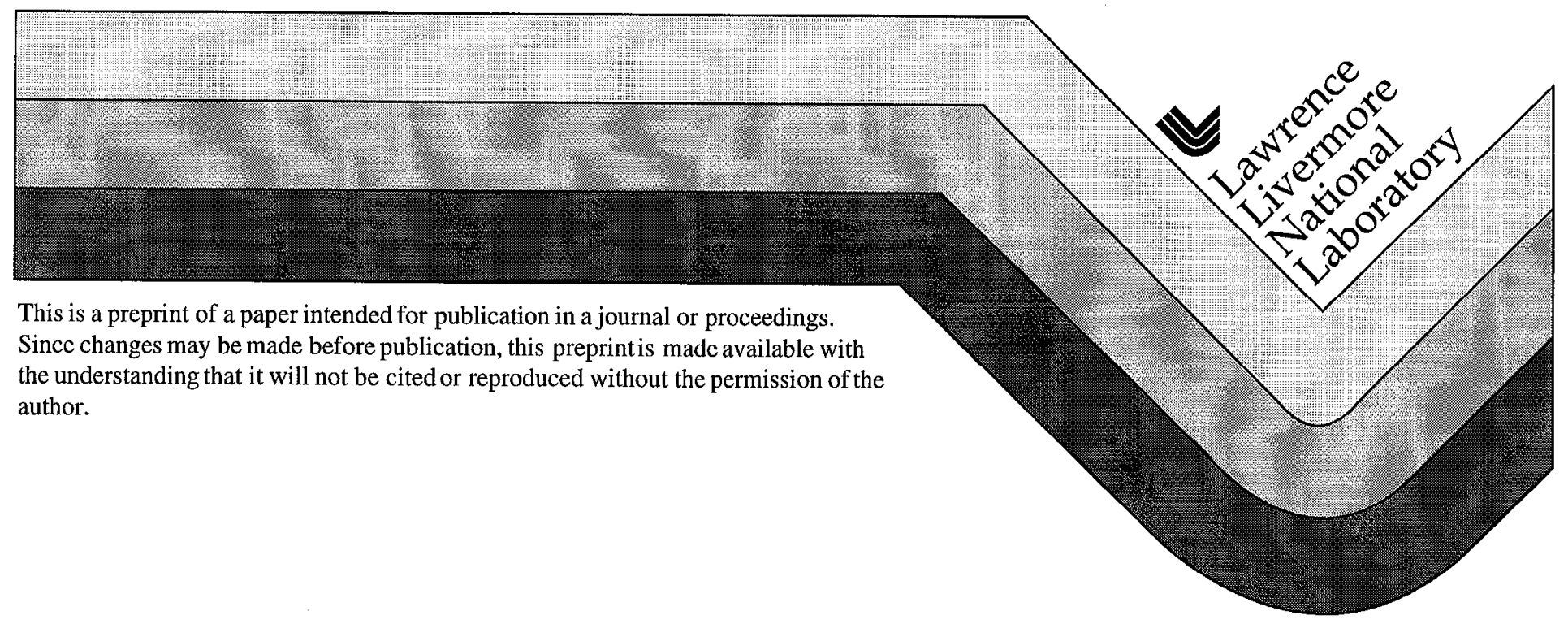




\section{DISCLAIMER}

This document was prepared as an account of work sponsored by an agency of the United States Government. Neither the United States Government nor the University of California nor any of their employees, makes any warranty, express or implied, or assumes any legal liability or responsibility for the accuracy, completeness, or usefulness of any information, apparatus, product, or process disclosed, or represents that its use would not infringe privately owned rights. Reference herein to any specific commercial product, process, or service by trade name, trademark, manufacturer, or otherwise, does not necessarily constitute or imply its endorsement, recommendation, or favoring by the United States Government or the University of California. The views and opinions of authors expressed herein do not necessarily state or reflect those of the United States Government or the University of California, and shall not be used for advertising or product endorsement purposes. 


\title{
Statistical description of laser damage initiation in NIF and LMJ Optics at $355 \mathrm{~nm}^{*}$
}

\author{
M.D. Feit, F.Y. Génin, A.M. Rubenchik, L.M. Sheehan, S. Schwartz and M.R. Kozlowski \\ Lawrence Livermore National Laboratory \\ mail stop $L-477$ \\ Livermore, CA 94550 USA \\ J. Dijon, P. Garrec and J. Hue \\ LETI-CEA-DOPT-Couches Minces pour l'Optique(CMO), CENG \\ 17, Avenue des Martyrs, 38054 Grenoble, Cedex 09 France
}

\begin{abstract}
Understanding the "extreme statistics" of failure at a weak link allows extrapolation of the results of small area laser damage tests to predict damage levels for the large areas pertinent to NIF/LMJ. Conceptually, it is important to focus on the fluence dependence of the surface density of damage sites. Results of different types of damage tests can be reported in terms of this sample characteristic property.
\end{abstract}

Keywords: laser initiated damage, statistics, large optics

\section{INTRODUCTION}

Laser induced surface damage initiates at extrinsic defects which affect the near-surface transparency. Qualitatively, this statement is supported by the probabilistic nature of measured damage thresholds and the increased variance of observed thresholds with decrease of the illuminated area. The specific nature of responsible defects doubtless depends on the material (glass, fused silica), polishing procedure, radiation wavelength, etc. In order to estimate operational damage probabilities for meter-size optics from experimental results with smaller optics, it is useful to apply a well founded phenomenological approach involving a few empirical parameters to do the extrapolation. This is the first step in developing a general laser damage reliability model for NIF/LMJ optics.

This problem is a special case of the general need to predict the reliability of a system subject to failure at its weakest points, a problem which has been of great importance to industry ${ }^{1}$. Specific examples of relevance concern the mechanical strength of optical fibers ${ }^{2}$ and the electrical breakdown of insulation on coaxial electrical cables ${ }^{3}$. In both of these cases, one wishes to estimate the behavior of very long cables from tests conducted on much shorter lengths. A similar approach was applied in [4] to studies of optical damage. In the present paper, we extend this approach to extrapolate small area damage test results to NIF/LMJ sized optics

The paper is organized as follows. In section II, we give a general conceptual overview of the problem of "extreme statistics", i.e. situations in which the overall strength is determined by the weakest point. The Weibull plot analysis is of general use in engineering and is discussed in ref.[1]. A summary of scaling laws determined from computational simulations of fracture and breakdown for this type of phenomena is given in refs.[5-6]. The reader is also referred to descriptions of damage threshold area scaling ${ }^{7}$ and the probabilistic analysis ${ }^{8}$ of damage in KDP. The third section discusses three case histories: a 1970's Arzamas study ${ }^{4}$ of area dependence of laser glass damage threshold, an analysis of a CMO 1000-site study of $3 \omega$ damage in fused silica and representative LLNL damage threshold data (S-curves) in the form most conductive to the type of analysis needed (Weibull plot). The fourth section of this paper points out the necessity of taking beamshape into account in extrapolating Gaussian beam tests to the NIF (flat beam) situation. The fifth section carries through a generic calculation of damage incidence using intensity statistics from LLNL's NIF prototype Beamlet laser. We extrapolate typical small sample damage test data to NIF/LMJ sized optics. This extrapolation gives encouraging results. Finally, we make several suggestions for gaining the necessary data and confidence to make reliable large area predictions of damage incidence. 


\section{EXTREME STATISTICS}

We will characterize the distribution of damage inducing defects by the fluence at which they cause damage. We define $P(S, F)$ to be the probability that a surface area $S$ exposed to radiation of increasing fluence will damage at fluence $F$. The complementary probability $U(S, F)=1-P(S, F)$ is then the probability to survive fluence $F$. Let the number of surface defects per unit area that produce damage at fluence between $F$ and $F+d F$ be defined as $n(F) d F$. Then, the incremental probability that the surface damages at $F+d F$ given that it did not damage at $F$ is the product of the probability of survival up to fluence $\mathrm{F}, \mathrm{U}(\mathrm{S}, \mathrm{F})$, times the probability of finding defects that damage in this interval, $\mathrm{nSdF}$. That is,

$$
P(S, F+d F)-P(S, F)=-(U(S, F+d F)-U(S, F))=S n(F) U(S, F) d F
$$

or in differential form

$$
\frac{d U(S, F)}{d F}=-S U(S, F) n(F)
$$

The probability to survive at fluence $\mathrm{F}$ is seen to be given by

$$
\left.U(S, F)=\exp \left[-S \int_{0}^{F} n\left(F^{\prime}\right) d F^{\prime}\right]\right] \equiv \exp [-S c(F)]
$$

Here $c(F)=\int^{F} n\left(F^{\prime}\right) d F^{\prime}$ is the cumulative areal density of defects that cause damage at fluence less than $F$.

It is convenient to normalize the distribution $n(F)$ in terms of the total number of defects/area $n$ by defining the fraction of defects that damage at fluence $F$, i.e. define $n(F)=n f(F)$ where $\int f(F) d F=1$.

To procecd further analytically, we must specify the distribution function $\mathrm{f}$. In many practical applications, a powcr law dependence of $f$ on the "stress" factor (here fluence F) has proved useful in analyzing experimental data. This so-called Wcibull distribution function refs.[1-2,4-5] is of widcsprcad use. A variation of this model was used by Arzamas researchers ${ }^{4}$ to analyze the area dependence of damage thresholds as discussed below. We will use the Weibull distribution in the following since it generally fits recent data for high quality fused silica.

Several features are immediately clear when the data follow a Weibull distribution. First, from Eq.(3), one sees that the area being tested appears in the exponent (this is true whether the concentration follows a power law or not). Thus, if the survival probability is $50 \%$ at a given fluence for a given laser spot size, increasing the beam area by a factor of 10 reduces the survival to $(1 / 2)^{10} \approx 0.1 \%$ at that fluence. Further, one can immediately extrapolate the results for one area to another using the same rule. However, this is not as useful as at first appears and the above is an over-pessimistic result for two reasons. First, the observed data have uncertainties including statistical sampling uncertainties and these increase proportionately with the increased area making the experimental results less useful at the larger area. Related to this is the fact that low probability low damage fluence events at small area become dominant for large areas, and these low probability events, of necessity, have large statistical uncertainties.

On the other hand, if one can reasonably fit an empirical model, such as the Weibull distribution, to the data and gain some confidence that it can be extrapolated to smaller fluences, then the model can be used to reliably estimate damage statistics for large areas. For example, suppose that one finds the cumulative defect distribution $\mathrm{c}(\mathrm{F})$ defined above to scale as the $\mathrm{m}^{\text {th }}$ power of fluence ( $\mathrm{m} \approx 7-13$ in LLNL data presented later). Then the $50 \%$ fluence for an area 10 times larger than that of the original experiment will be reduced by a factor of $10^{1 / \mathrm{m}}$ which is a reduction of only about $20 \%$. The fact that both statements can be true (survival at original fluence is near zero, $50 \%$ fluence drops by $20 \%$ ) is a reflection of the fact that the cumulative damage probahility curve (S-curve) becomes sharper for larger areas.

\section{CASE HISTORIES}

The experiments reported in ref.[4] used 1 micron wavelength (IR) laser pulses of $100 \mathrm{~ns}$ duration on K-8 glass. Of course, this is a different glass which underwent different finishing than NIF/LMJ optics. Nevertheless, it is instructive to discuss their results and interpretations. It is also important to note that they reported strong effects of conditioning. 
The cumulative defect density $c(F)$ of ref[4] was found to vary as the $m=2.5$ power of fluence. It is clear from the discussion at the end of the introduction that one prefers as large a value of $m$ as possible. Indeed, we will see below that good optics have $\mathrm{m}$ as high as 14 , and thus appear to be much more defect free than the glass tested in this study.

We have carried out two types of damage tests. The Automatic Damage Test (ADT) increases the fluence (R/1 test) until a site damages. A cumulative damage probability curve is found from the ratio of the number of sites that damage below fluence $\mathrm{F}$ to the total number of sites. The Large Area Test (LAT) raster scans an area at constant fluence leading to an area density of damage sites.

An approximately 1000 sites ADT study of $3 \omega$ damage on polished fused silica was carried out earlier ${ }^{9}$. Results presented here are scaled to $3 \mathrm{~ns}$ pulse duration. We make two points from the earlier results. First, because of the large number of sites, we can investigate how the number of sites tested influences the conclusions inferred from the experiment. Secondly, the statistical distribution appears somewhat different than that of the current best LLNL optics and is another indication of the progress made in the past year or so in improving the damage resistance of optical materials.

Damage testing results usually are presented in terms of the cumulative damage S-curve. The actual observations from which the S-curve is derived typically show considerable statistical scatter. We show in Fig.(1) the $1 \mathrm{~mm}^{2}$ damage probability distributions derived from 100, 500 and 900 sites samples from the 1000 sites data. Although the average damage threshold value of $19.3 \mathrm{~J} / \mathrm{cm}^{2}$ is accurately given by the 100 site sample, there is variation in the maximum and minimum fluences found with the population size. Thus, it is the outlying, low probability points that are most uncertain as one would expect. It is, of course, precisely the low fluence low probability values that are needed for extrapolation to large areas. Fig.(2) shows a Weibull plot $(\ln (-\ln (1-\mathrm{P}))$ vs. $\ln \mathrm{F})$ for this data. Note that the addition of more sites affects mainly the lower part of the curve. Overall, this data does not fit the Weibull form nearly as well as more recent ADT and Raster Scan data. This may be a clue about overlapping distributions of different kinds of defects in the earlier materials.

For experimental data processing, it is useful to plot not only the cumulative damage probability vs. fluence (Scurve), but also the quantity $\mathrm{N}=-\ln U(\mathrm{~S}, \mathrm{~F})$ vs $\mathrm{F}$, i.e. the Weibull plot. In Weibull analysis, $\mathrm{N}$ is assumed proportional to the cumulative defect density, ie. the total number of defects per unit area damaged at fluences up to $\mathrm{F}$. The density derived this way is independent of the measurement area (assuming sample homogeneous) and is characteristic of the test sample. A typical Weibull distribution appears linear on a log-log plot.

Experimentally, in ADT testing, the total test area is divided into many small area sites and the probability distribution found refers to the single site area. At each site, the laser intensity ramps up step by step up until the site damages. Such measurements of damage probability assume the absence of conditioning(decreased damage vulnerability due to low fluence shots) and contamination from previously damaged sites.

We illustrate both typical S-curves and the corresponding Weibull plots for ADT results in fused silica prepared with differing finishing processes Figs. $(3,4)$.

In Fig.(4), the main part of all but one of the distributions fits a power law with Weibull index $m=10-12$. The exception is one sample where $m$ is about hall as large. A parallel shift of the defect concentration can be interpreted as a change in the total number of defects for the same normalized distribution function $\mathrm{f}$. All the curves, similar at high fluence, have differences at low fluence due, at least in part, to the small amount of data available at lower fluences. The same pattern is seen for the fused silica data shown in Fig(5) corresponding to a different finishing process and performed with different test equipment.

A power law distribution indicates the absence of any special spatial scale for the defects such as occurs, for cxamplc, for an exponential distribution. The distribution contains information about the underlying damage mechanism. Consider a specific model of responsible defects, e.g. absorbing inclusions ${ }^{10}$. In this case, the temperature near the inclusion is proportional to the inclusion radius a, laser fluence $\mathrm{F}$ and absorption efficiency $\alpha$. Damage is assumed to occur when the tcmpcrature reaches some critical value. We can conclude that if inclusions larger than the laser wavelength $(\alpha$ is independent of a) were responsible for damage, then the concentration of inclusions with size a would vary as $1 / \mathrm{a}^{\mathrm{m}}$. If the inclusions are smaller than the wavelength ( $\boldsymbol{\alpha}$ is proportional to a), the defect concentration varies as $1 / \mathrm{a}^{2 \mathrm{~m}}$. It is difficult to rationalize how such a distribution might occur. 
The situation is quite different for defects characterized by their length such as cracks. Suppose the density of cracks is a function of length $L$, say $1 / L^{q}$. The damage threshold may depend on cracklength, say as $1 / L^{p}$. In this case, the Weibull index is found to be $m=(q-1) / p-1$. One can see that for small $p$, the value of $m$ can be large even if $q$ is not.

If all cracks have similar widths, damage fluence is expected to be only weakly dependent on length. Thus, the effective value of $p$ will be small and $m$ will be large. Thus, the experimental data is consistent with the conclusion that most damage on high quality samples originates in small subsurface cracks.

The low fluence tail of the damage probability curve (Figs(3-6)) changes from sample to sample. It is not clear to what extent this is due to statistical fluctuations, unique (non-statistical) random defects produced by handling and processing or all of the above. More observations at low fluence are desirable to elucidate this point.

Eq.(3) has an explicit area dependence. Even without knowledge of the defect distribution function, the raw data can be extrapolated to larger areas with the reservations discussed above. Fig.(7) plots an original S-curve and its extrapolation to areas 5 and 10 times larger than the experimental value. One sees that rescaling to larger areas requires more detailed knowledge of the low end of the damage probability curve. However, as discussed above, experimental data are scarce here and statistical fluctuations are significant. It should also be noted that experimental uncertainties are magnified when rescaling to larger area. For small uncertainties $\Delta \mathrm{P}<<\mathrm{P}$, scaling from area $\mathrm{S}$ to $\mathrm{S}_{1}$ implies $\Delta \mathrm{P}\left(\mathrm{S}_{1}\right)=\left(\mathrm{S}_{1} / \mathrm{S}\right) \Delta \mathrm{P}(\mathrm{S})$.

In ref. [4], the authors used a power law to fit the cumulative defect density $c(F)$ to experimental data for large areas and found good agreement with experimental results. Direct rescaling of data would be much less reliable for the reasons presented above.

Fig.(8) presents calculated damage probability S-curves for three different test areas assuming the 50\% damage probability fluence is $20 \mathrm{~J} / \mathrm{cm}^{2}$ for a $1 \mathrm{~mm}^{2}$ beam. The $S$-curve has a steep slope $(\mathrm{m}=11)$, which shifts to lower fluence as the area increases according to Eq.(3). For large $\mathrm{m}$, this shift is not very sensitive to the area increase as noted above. For example, for $\mathrm{m}=11$, an increase of area 100 times decreases the threshold fluence only by $35 \%$ (Fig.(8)). It should be noted that in this case lower values of the fluence are most important.

\section{IMPORTANCE OF DAMAGE TEST BEAMSHAPE AND SIZE}

The above refers to the "illuminated area", but one has to be careful about what this means for a nonuniform beam. In particular, we have seen from the above that damage probability for high quality samples typically depends on a high power of the fluence, e.g. $m=12$. Fluence raised to the $m^{\text {th }}$ power is much more sharply peaked than the fluence itself. In general, one should replace $S \mathrm{c}(\mathrm{F})$ in Eq.(3) by the integral $\int \mathrm{c} \mathrm{dx} d y=c\left(F_{0}\right) S_{\text {eff }}$, where $\mathrm{F}_{0}$ is the peak fluence. Thus for a Gaussian beam with $\mathrm{F}=\mathrm{F}_{0} \exp \left(-(\mathrm{r} / \sigma)^{2}\right)$, one finds the appropriate area to use is $\pi \sigma^{2} / \mathrm{m}$ in order to find the "intrinsic" damaging defect density needed to estimate results for a flat beam. Thus, an effective area, determined jointly by the beamshape and the defect density must be calculated to normalize the defect density found from experimental observations.

In a typical small beam area damage test, measurable damage is generally found only at fluences greater than operational fluences for large area optics. The Weibull distribution (once ascertained correct) can be used to scale the results to lower fluences.

As an example, consider recent ADT tests ( $7.5 \mathrm{~ns}$ pulse) on a Zirconia polished sample. The beam $1 / \mathrm{e}^{2}$ radius was 1 $\mathrm{mm}$ so the effective area is about $0.1 \mathrm{~mm}^{2}$. The experimentally determined cumulative densities at $36 \mathrm{~J} / \mathrm{cm}^{2}$ were found to be $c_{1}\left(36 \mathrm{~J} / \mathrm{cm}^{-2}\right)=411 \mathrm{~cm}^{-2}$ with $\mathrm{m}=17$ and $\mathrm{c}_{2}\left(36 \mathrm{~J} / \mathrm{cm}^{2}\right)=376 \mathrm{~cm}^{-2}$ with $\mathrm{m}=14$ where the subscripts refer to side 1 and side 2 . The NIF redline for $7.5 \mathrm{~ns}$ pulses is $20.6 \mathrm{~J} / \mathrm{cm}^{2}$. At this fluence, the damaging defect density is expected to be given by $\mathrm{c}(36)$ $(20.6 / 36)^{\mathrm{m}}$ which gives $c_{1}=0.03 \mathrm{~cm}^{-2}$ and $c_{2}=0.15 \mathrm{~cm}^{-2}$. For a NIF size optic of $1600 \mathrm{~cm}^{2}$ area, we thus expect about 48 damage sites on side 1 and 240 sites on side 2 at the redline fluence. The difference is due to the different values of $\mathrm{m}$ (see Fig.(9) ). The low values reflect the high damage resistance quality of the samplc. The extrapolated differences for the large area emphasize the utility of using the exponent $m$ as a figure of merit. At the lower operational fluences, very few damage sites would be expected on a largc optic made of this material. 
The analysis presented here allows comparison between different types of tests, eg. the ADT tests shown above and large area raster scans (LAT) in which case tens of $\mathrm{cm}^{2}$ are scanned at constant fluence and resulting damage sites counted. For raster scans, the effective area defined in the beginning of this section depends on the ratio of Gaussian beam size to scan step size. Fig.(6) compares ADT and LAT tests for silica with various polishing processes. It is seen that the cumulative damaging defect densities found cover a range spanning six orders of magnitude.

\section{GENERIC CALCULATION OF EXPECTED DAMAGE S-CURVE FOR NIF SIZED OPTIC}

Above, we considered the probability of finding a single damage site within an area S. For a very large area, the practical issue is the damage density or area per damage site given some fluence distribution. Thus, the goal of small sample damage tests is to predict the damage density on a very large sample. The observed results presented above suggest the following approach.

Typical R/1 tests yield damage probability for an effective flattop beam area given by the beamsize and the slope of the Weibull curve, i.e. $\pi \sigma^{2} / \mathrm{m}$. For a sufficiently large population of sites, especially at the lower fluences, one can use the power law fit to extrapolate to the lowest fluences of operational interest. Large area beams never have uniform fluence, but rather some distribution (Fig.(10)). For example, for a beam with normal, ie. Gaussian beam statistics, one can make a simple analytic estimate of the effect of the fluence distribution. The expectation value of the exponent in Eq.(3) can be found by expanding the integrand around the mean fluence value. If the average fluence is $F_{0}$ and the $1 / e$ width of the tluence distribution is $\Delta \mathrm{F}$, then one finds

$\mathrm{P}\left(\mathrm{F}_{0}\right)=1-\exp \left(-\mathrm{S}<\mathrm{c}\left(\mathrm{F}_{0}\right)>\right)$, where

$$
<c\left(F_{0}\right)>\approx c\left(F_{0}\right) \sqrt{\frac{4 b^{2}}{4 b^{2}+2 m}} \operatorname{Exp}\left[\frac{m^{2}}{4 b^{2}+2 m}\right]
$$

Here, $\mathrm{P}\left(\mathrm{F}_{0}\right)$ is the expected damage probability as a function of the mean fluence, $\mathrm{P}_{0}=1-\exp \left(-\mathrm{S} c\left(\mathrm{~F}_{0}\right)\right)$ is the damage probability obtained for a perfect flattop beam at fluence $\mathrm{F}_{0}, \mathrm{~b}=\mathrm{F}_{0} / \Delta \mathrm{F}$, and $\mathrm{m}$ is the slope of the Weibull curve. This is a good approximation provided that

$$
\mathrm{b}=\mathrm{F}_{0} / \Delta \mathrm{F}>\mathrm{m}^{1 / 3}
$$

i.e. for a sufficiently small variance (e.g. for $\mathrm{m}=12, \Delta \mathrm{F} / \mathrm{F}_{0}$ should be less than 0.4$)$. Here $\mathrm{c}(\mathrm{F})$ is the cumulative damage density as before and $\langle\mathrm{c}>$ refers to the expectation value. Eq.(4) gives insight into the effect of fluence deviations. Eq.(4) is approximate; in the rare case where $m$ does not satisfy Eq.(5), it is possible to simply find $\mathrm{P}\left(\mathrm{F}_{0}\right)$ by numerical integration using the exact expression

$$
<c\left(F_{0}\right)>=\int_{0}^{\infty} c(F) f(F) d F
$$

where $f(F)$ is the fluence distribution and the Weibull fit gives the cumulative defect density $c(F)$.

We present here "generic" type calculations to show the orders of magnitude involved. Fig. (10) shows a typical experimental fluence distribution at a large lens on the LLNL Beamlet laser. The mean $3 \omega$ fluence is $5.3 \mathrm{~J} / \mathrm{cm}^{2}$ and the full $1 / \mathrm{e}$ width is about $30 \%$. Fig.(9) shows the result of extrapolating small area results for typical high quality damage samples (damage probability $50 \%$ at $30 \mathrm{~J} / \mathrm{cm}^{2}$ for $1 \mathrm{~mm}^{2}$ beam area) to a $1600 \mathrm{~cm}^{2}$ area . S-curves for both $\mathrm{m}=12$ and $\mathrm{m}=14$ are shown for comparison; the advantage of large Weibull slope $m$ is evident. Note that the better value shows damage just beginning on the large area at the NIF relevant $8 \mathrm{~J} / \mathrm{cm}^{2}$. In Fig.(11), we plot the change in predicted damage probability found from Eq.(6), ie. $P\left(F_{0}\right)-P_{0}$ due to the fluence distribution of Fig.(9) for $m=12$. As cxpected, the shift in the S-curve duc to this effect is small.

\section{CONCLUSIONS}

Reliable probabilistic estimates of damage for large optics can be based on damage studies of smaller samples. Conceptually, it is important to focus attention not on the probability of damage which depends on the size of the area tested, but, rather, on the areal density of sites that damage at a given fluence. This density is characteristic of a homogenous material and can be extrapolated to find the damage density on an infinite area sample. The practical requirement for such 
extrapolation is adequate knowledge of the low fluence damaging defect density $c(\mathrm{~F})$. The following recommendations and questions are suggested from the above.

Previous treatments of laser damage tried to establish a threshold for a particular sample. The statistical nature of damage has been recognized by introducing the threshold distribution (S-curve) for a given sample. In practical terms, the relevant question is what is the probability for a given tolerable damage density. Reliable estimates of this probability will depend on accurate knowledge of the damaging defect densities described above. The tolerable level of damage is likely to be different for different materials and operating environment. For example, $1 \omega$ spatial filter lenses with one side in vacuum and the other side in air are subject to large mechanical loading. Since the more vulnerable $3 \omega$ optics are in vacuum and at the end of the chain, the level of tolerable damage can be quite different.

Reliable probabilistic estimates of damage levels will require not only extrapolating small area damage observations to larger areas, but also knowledge of how initial pinpoint damage grows with subsequent shots. This knowledge can then be combined with a detailed model of temporal and spatial fluctuations expected in the NIF pulse to produce realistic estimates. Of course, one cannot be absolutely sure there are no "extremely rare" damaging defects without full aperture scanning.

*Work performed at LLNL under the auspices of the U.S. Department of Energy under contract No. W-7405-ENG-48.

\section{REFERENCES}

1. S. Zacks, Introduction to reliability analysis. Probability models and statistical methods, (Springer-Verlag, New York, 1992).

2. R. Olshansky and R.D. Maurer, "Mechanical strength and fatigue of optical fibers", J. of Appl.Phys. 47, 4497, (1976).

3. 1. Simoni, G. Mazzanti, G. Montano, "Electrical breakdown of insulating materials and structures" in Size-scale Effects in the Failure Mechanisms of Materials and Structures, edited by A. Carpinteri, (E\&FN Spon, Chapman and Hall, London, 1996).

4. A.V. Bessarab et al, "Statistical relationship governing the surface damage of optical glass by wide laser radiation beams", Sov. J. Quantum Electron 7,181, (1977).

5. B.K. Chakrabarti and L.G. Benguigui, Statistical physics of fracture and breakdown in disordered systems, (Clarendon Press, Oxford, 1997).

6. I.C. Van den Born, A. Santen, H.D. Hoekstra and J. De Hossen, "Mechanical strength of highly porous ceramics", Phys. Rev. B 43, 3794 (1991).

7. J. Hue, F. Y. Génin, S. M. Maricle, and M. R. Kozlowski, "Toward predicting the laser damage threshold of large-area optics", Laser-induced damage in optical materials, SPIE - 2966, 451 (1996).

8. M. Runkel, "Overview of recent KDP damage experiments and implications for NIF tripler performance", (in these proceedings).

9. F.Y. Génin, L. Sheehan, J. Yoshiyama, J. Dijon and P. Garrec, "Statistical study of UV laser induced failure of fused silica", Laser Induced Damage in Optical Materials 1997, SPIE-3244, 155-63 (1998).

10 . M.D. Feit, J. Campbell, D. Faux, F.Y. Génin, M.R. Kozlowski, A.M. Rubenchik, R. Riddle, A. Salleo and J. Yoshiyama, "Modeling of laser induced surface cracks in silica at $355 \mathrm{~nm}$ ", Laser Induced Damage in Optical Materials 1997, SPIE-3244, 350-3 (1998). 


\section{Figures}

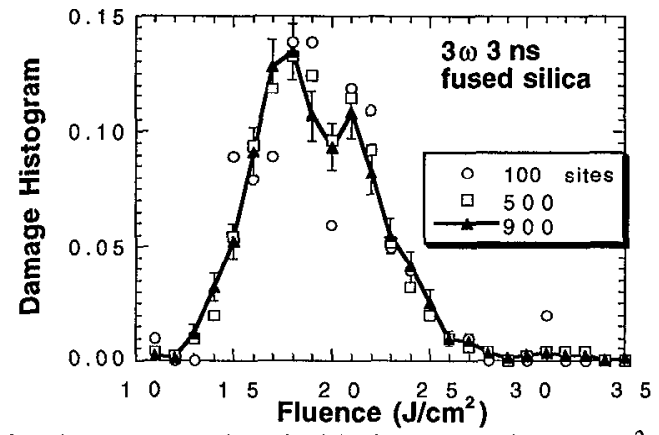

Fig. 1: Damage threshold histogram for $1 \mathrm{~mm}^{2}$ of polished fused silica. Results for 100, 500 and 900 sites are shown. All ADT tests refer to $1 \mathrm{~mm}^{2}$ beam.

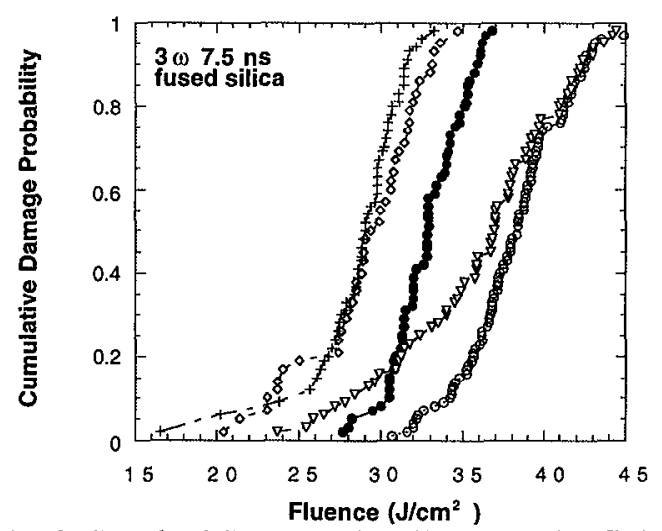

Fig. 3: Standard S-curves for silica samples finished with three different polishing processes. $\tau=7.5 \mathrm{~ns}$.

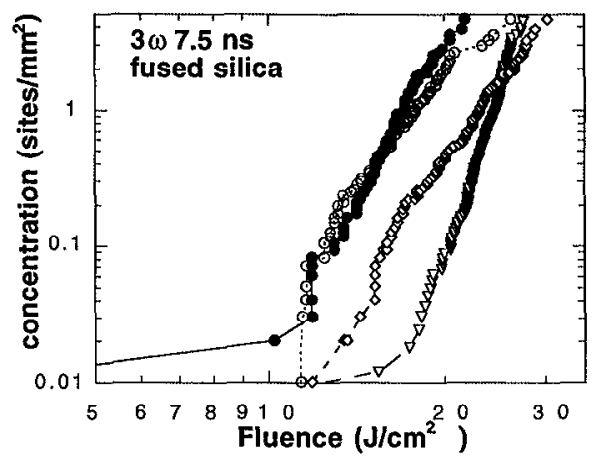

Fig. 5: Cumulative damaging defect distribution functions for fused silica samples with another polishing process. $\tau=3 \mathrm{~ns}$.

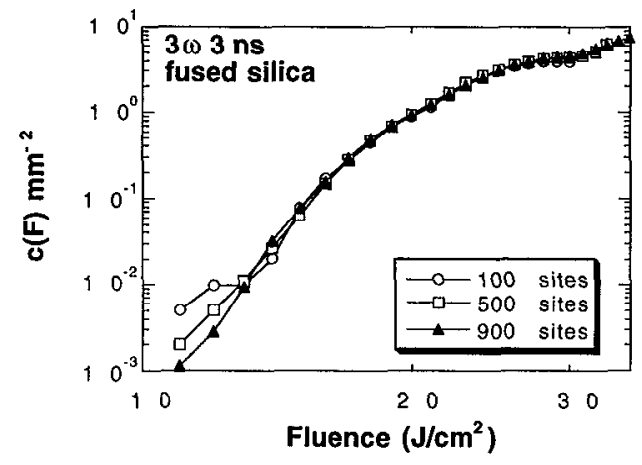

Fig. 2: Weibull plot for data of Fig.(1). The cumulative damage density is given as a function of fluence.

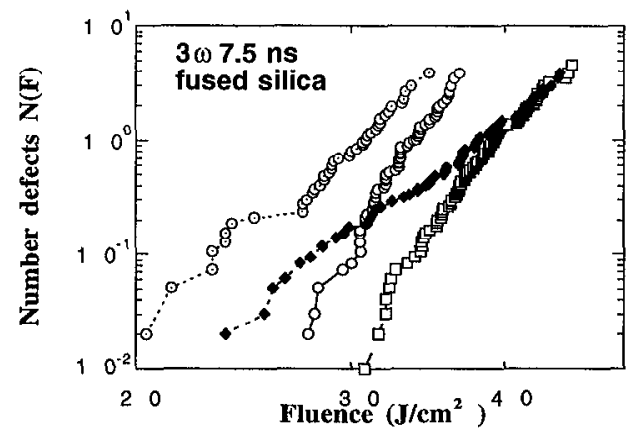

Fig. 4: Cumulative damaging defect distribution functions found from the data in Fig.(3).

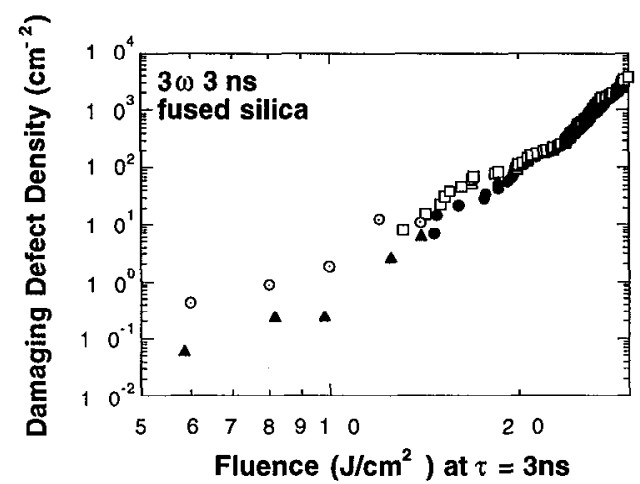

Fig.6: Comparison of results from two ADT tests at different areas of fused silica with one polishing process (upper right) with results of two LAT raster scans of fused silica with two other polishing processes (lower left). 


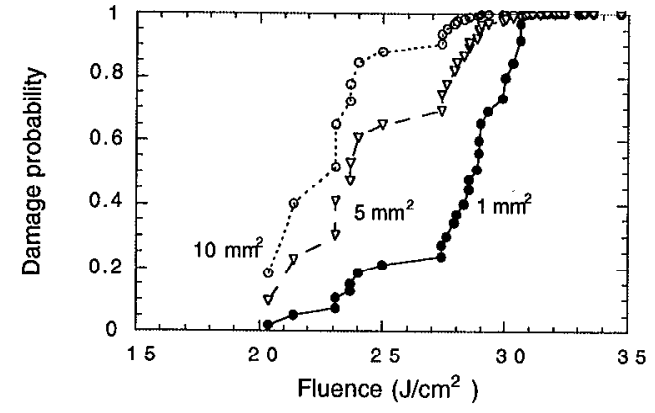

Fig.7: Original S-curve with rescaling to areas 5 and 10 times larger. Note error bars on last curve are 10 times as large as on experimental points.

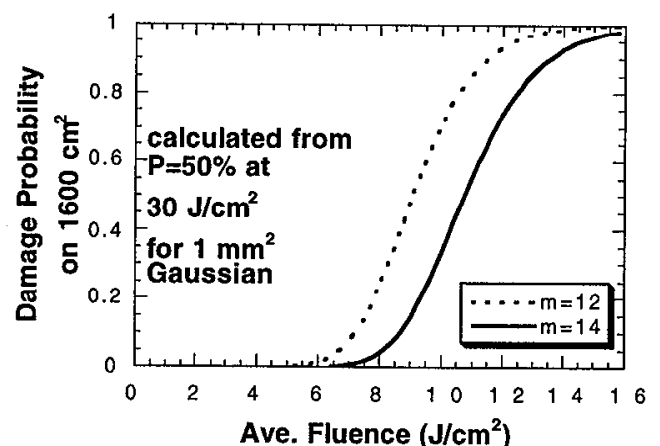

Fig. 9: Calculated damage S-curves for area of 1600 $\mathrm{cm}^{2}$ illuminated by beam with distribution given in Fig.(10) and extrapolating Weibull expression corresponding to $50 \%$ damage probability at 30 $\mathrm{J} / \mathrm{cm}^{2}$ for $1 \mathrm{~mm}^{2}$ Gaussian test beam.

\section{$\Delta \mathrm{P}$}

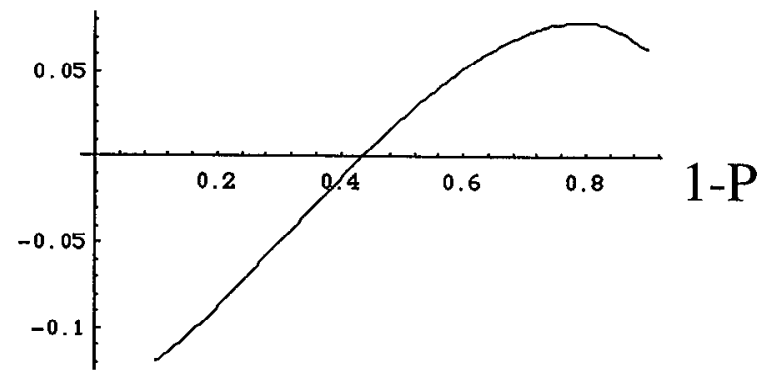

Fig. 11 Deviation of calculated damage probability curve corresponding to fluence distribution shown in Fig.(10) from that of perfect flattop beam for $m=12$.

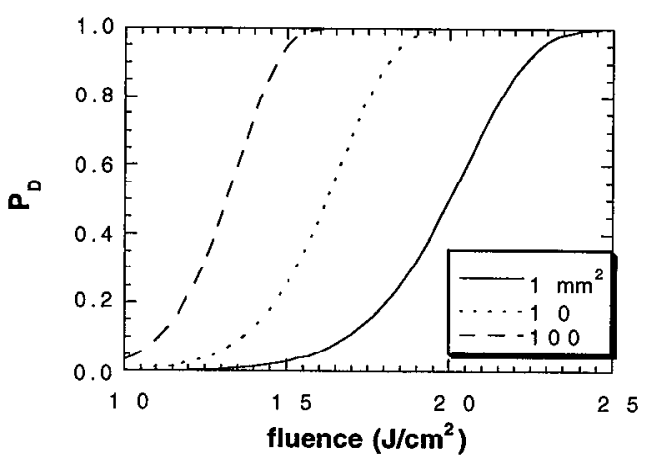

Fig. 8: Damage probability curves calculated from theory assuming $50 \%$ probability at $\mathrm{F}=20 \mathrm{~J} / \mathrm{cm}^{2}$ for area of $1 \mathrm{~mm}^{2}$. Results shown for 3 different areas assuming Weibull exponent $m=11$.

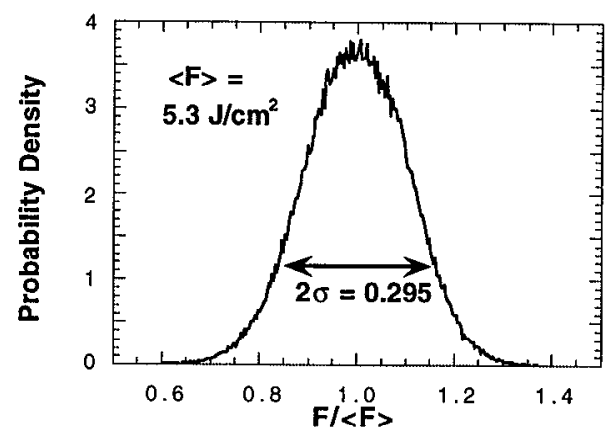

Fig. 10: Typical Beamlet intensity distribution (courtesy of $\mathrm{P}$. Wegner) for medium damage threshold campaign with average fluence of 5.3 $\mathrm{J} / \mathrm{cm}^{2}$. 